\title{
Predisposition to thrombosis not reflected by the blood coagulogram
}

\author{
B. SOLYMOSS, H. SELYE, AND G. GABBIANI \\ From the Institut de Médecine et de Chirurgie expérimentales, Université de Montréal, \\ Montreal, Canada
}

SYNOPSIS Experiments on rats indicate that thrombosis of large veins can develop while blood coagulability is decreased. Hence, coagulation tests have only a limited value in predicting a thrombotic tendency, although they do show characteristic changes during the formation of thrombi.

According to Virchow three major factors play a role in thrombus formation: (1) deterioration of the vessel wall; (2) circulatory changes; and (3) changes of the blood composition (Virchow, 1856). After more than a century of intensive study this view is still accepted today (O'Neill, 1947; Wessler, 1963; Zweifach, 1963). However, despite many efforts to identify premonitory changes in blood coagulation factors (Pechet and Alexander, 1961; Egeberg and Owren, 1963; Wessler, 1963) a reliable method for the demonstration of a thrombotic tendency has not yet been found (Evans, 1954; Quick, 1958). This lack of success may be due to the fact that red thrombi can develop as a consequence of stasis, sludging, and hypercoagulability (Wessler, 1963), but in the formation of white thrombi, vessel wall deterioration followed by thrombocyte adhesion and aggregation appear to play a more important role (Rodman, Mason, and Brinkhous, 1963) and these are not revealed by coagulation studies.

In view of the considerable amount of work performed in efforts to correlate predisposition to thrombosis with blood coagulation changes, we would like to report here observations demonstrating the possibility of thrombus formation during the state of hypocoagulability, thereby casting doubt on coagulation tests purporting to reveal a thrombotic tendency.

Previous studies have shown that a thrombohaemorrhagic phenomenon can be elicited, for example, in the rat, by a dual treatment: (1) systemic administration of various 'sensitizers' (in most instances metals or polysaccharides) which produce a general thrombo-haemorrhagic diathesis; (2) topical or systemic treatment with certain 'challengers', e.g., catecholamines, serotonin, vasopressin, or mastcell dischargers, which make this latent disease Received for publication 12 March 1966. proneness manifest and determine the localization of the resulting lesions. Using the thrombo-haemorrhagic phenomenon techniques, it has even been possible to produce thromboses in such large vessels as the iliac veins or the vena cava inferior (Selye and Tuchweber, 1964; Selye, Tuchweber, and Rohan, 1965; Gabbiani, Jacqmin, and Selye, 1966). Interestingly, several metals which induce susceptibility to the thrombo-haemorrhagic phenomenon also have an anticoagulant effect; hence we studied blood coagulation during the development of femoral-vein thrombi induced by one of the above-mentioned methods (Selye et al., 1965).

\section{MATERIALS AND METHODS}

One hundred and sixty-five female Sprague-Dawley rats with an initial body weight of $206 \mathrm{~g}$. (196-211 g.) were divided into 15 groups for two experiments. In the first of these (Table I) we examined the action of scandium chloride ${ }^{1}$ on blood coagulation in vitro. In two groups of 10 animals each, $4.5 \mathrm{ml}$. of blood was taken by aortic puncture and mixed in a ratio of $9: 1$ with $1.34 \%$ sodium oxalate in a siliconized tube. To each tube we added $1 \mathrm{ml}$. of distilled water in the first group and $6 \mathrm{mg}$. of scandium chloride in $1 \mathrm{ml}$. of distilled water in the second group. The second experiment (Tables II and III) was performed in vivo. Because of the high mortality, groups $4,7,10$, and 13 consisted of $11,12,14$, and 18 rats respectively, the others of 10 rats. The first group received $1 \mathrm{ml}$. of distilled water intravenously and served as control. The animals of groups $2,5,8$, and 11 received adrenaline bitartrate $^{2}$ at a dose of $30 \mu \mathrm{g}$. in $0.1 \mathrm{ml}$. of distilled water intramuscularly close to the left femoral vein after incision of the skin. The animals of groups 3, 6, 9, and 12 received $6 \mathrm{mg}$. of scandium chloride in $1 \mathrm{ml}$. of distilled water intravenously and those of groups $4,7,10$, and 13 were, in addition, injected immediately thereafter with adrenaline bitartrate at the same dose and route as

${ }^{1} \mathrm{ScCl}_{3} \mathrm{~K}$ and $\mathrm{K}$ Laboratory, Plainview, N.Y., U.S.A.

${ }^{2}$ Brickman and Co. Ltd., Montreal, Canada. 
previously described. All manipulations were performed under light ether anaesthesia. The different groups were killed at different times and the means and standard errors ( \pm S.E.) of the results tabulated (Tables II and III).

Immediately after taking the blood by aortic puncture, we examined the thromboplastin generation (Sirridge, Bowman, and Allwin, 1962); recalcification time (Owen, Mann, Hurn, and Stickney, 1955); partial thromboplastin time (Langdell, Wagner, and Brinkhous, 1953) with Platelin ${ }^{3}$; prothromtin content (Quick, 1935) with Calsoplastin ${ }^{4}$; thrombin time (Perlick, 1960) (Table II); heparin tolerance (Soulier, 1951) with Liquaemin sodium $^{5}$; fibrinogen content (Perlick, 1960); thrombocyte (Perlick, 1960) and leucocyte count (Table III).

\section{RESULTS}

As seen from Table I scandium chloride delays blood coagulation in vitro. In its presence there is a decrease of thromboplastin generation $(P<0.001)$, prothrombin content $(\mathrm{P}<0.001)$ and heparin tolerance $(P<0.001)$, and an increase of recalcification $(\mathrm{P}<0.001)$, partial thromboplastin and thrombin times $(P<\mathbf{0 . 0 0 1})$.

As seen from Tables II and III adrenaline alone increased prothrombin content $(P<0.001)$ and thrombocyte count $(P<0.001)$ at all time intervals. Two, four, and 24 hours after the adrenaline administration there was an increase in partial thromboplastin time $(P<0.005)$. The fibrinogen content decreased one hour after treatment $(P<0.005)$, but 24 hours after was increased $(P<0.001)$. No thrombi were found in the animals injected with adrenaline alone.

One, two, and four hours after the intravenous

'Warner, Chilcott, Toronto, Ont., Canada.

'Baltimore, Bact. Laboratory, Baltimore, Md., U.S.A.

sOrganon Inc., West Orange, N.J., U.S.A.
TABLE I

IN VITRO EFFECT OF SCANDIUM CHLORIDE ON BLOOD COAGULATION

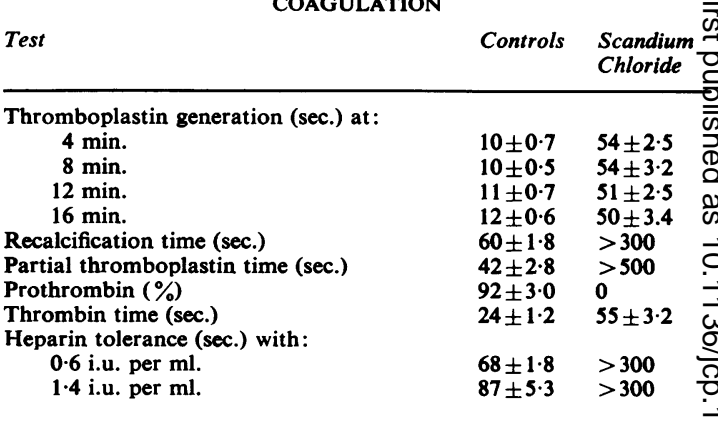

injection of scandium chloride there was an increaseof recalcification $(P<0.001)$, partial thrombow plastin $(P<0.001)$ and thrombin times $(P<0.001)$ and a decrease in prothrombin content $(P<0.001)$ ? The thromboplastin generation screening test $\mathrm{re}_{\overrightarrow{\underline{C}}}$ vealed a slight change in the substrate time aftes eight minutes of incubation $(P<0.005)$. Twenty four hours later all the above-mentioned changes returned to normal (Table II). Table III shows that one, two, and four hours after scandium chloride there was a decrease in heparin tolerance ( $P$ 0.001 ); after 24 hours there was a small but signio ficant decrease in the thrombocyte count $(P<0.001 \stackrel{\text { D }}{ }$ and an increase of fibrinogen concentration $(P \&$ $0 \cdot 001$ ). No thrombus formation was found in the animals treated with scandium chloride alone Adrenaline aggravated the blood coagulation changes produced by scandium chloride withoup altering their character. In the groups killed aftes four hours there was a significant difference in thromboplastin generation $(P<\mathbf{0 . 0 0 1})$, prothrombing

TABLE II

IN VIVO EFFECT OF SCANDIUM CHLORIDE AND ADRENALINE ON BLOOD COAGULABILITY

\begin{tabular}{|c|c|c|c|c|c|c|c|c|c|c|}
\hline \multirow[t]{2}{*}{ Group } & \multirow[t]{2}{*}{ Treatment } & \multirow{2}{*}{$\begin{array}{l}\text { Time of } \\
\text { Deter- } \\
\text { mination } \\
(\text { hr. })\end{array}$} & \multicolumn{4}{|c|}{ Thromboplastin Generation (sec.) } & \multirow{2}{*}{$\begin{array}{l}\text { Recalcifi- } \\
\text { cation } \\
\text { Time } \\
\text { (sec.) }\end{array}$} & \multirow{2}{*}{$\begin{array}{l}\text { Partial } \\
\text { Thrombo- } \\
\text { plastin } \\
\text { Time } \\
\text { (sec.) }\end{array}$} & \multirow{2}{*}{$\begin{array}{l}\text { Prothrom- } \\
\text { bin } \\
\text { Content } \\
(\%)\end{array}$} & \multirow{2}{*}{$\begin{array}{l}\text { Thrombin } \\
\text { Time } \\
\text { (sec.) }\end{array}$} \\
\hline & & & 4 Min. & 8 Min. & $12 \mathrm{Min}$ & $16 \mathrm{Min}$. & & & & \\
\hline 1 & None & - & $10 \pm 0.5$ & $10 \pm 0.4$ & $11 \pm 0.6$ & $11 \pm 0 \cdot 3$ & $67 \pm 6 \cdot 5$ & $41 \pm 1 \cdot 9$ & $96 \pm 2 \cdot 0$ & $19 \pm 0.3$ \\
\hline $\begin{array}{l}2 \\
3 \\
4\end{array}$ & $\begin{array}{l}\text { Adrenaline } \\
\mathrm{ScCl}_{3} \\
\mathrm{ScCl}_{3}+\text { adrenaline }\end{array}$ & $\begin{array}{l}1 \\
1 \\
1\end{array}$ & $\begin{array}{r}9 \pm 0.3 \\
13 \pm 0.4 \\
12 \pm 0.4\end{array}$ & $\begin{array}{l}10 \pm 0.5 \\
12 \pm 0.6 \\
13 \pm 0.3\end{array}$ & $\begin{array}{l}12 \pm 0.5 \\
13 \pm 0.6 \\
14 \pm 0.3\end{array}$ & $\begin{array}{l}13 \pm 0.6 \\
15 \pm 0.4 \\
15 \pm 0.4\end{array}$ & $\begin{array}{c}64 \pm 1 \cdot 7 \\
293 \pm 30 \cdot 5 \\
239 \pm 19 \cdot 0\end{array}$ & $\begin{array}{r}41 \pm 1 \cdot 9 \\
98 \pm 6 \cdot 0 \\
103 \pm 8 \cdot 4\end{array}$ & $\begin{array}{r}110 \pm 2 \cdot 0 \\
50 \pm 7 \cdot 0 \\
44 \pm 4 \cdot 0\end{array}$ & $\begin{array}{l}21 \pm 0.6 \\
>100 \\
>100\end{array}$ \\
\hline $\begin{array}{l}5 \\
6 \\
7\end{array}$ & $\begin{array}{l}\text { Adrenaline } \\
\mathrm{ScCl}_{3} \\
\mathrm{ScCl}_{3}+\text { adrenaline }\end{array}$ & $\begin{array}{l}2 \\
2 \\
2\end{array}$ & $\begin{array}{l}10 \pm 0.4 \\
10 \pm 0.3 \\
13 \pm 0.5\end{array}$ & $\begin{array}{l}11 \pm 0 \cdot 2 \\
11 \pm 0 \cdot 2 \\
13 \pm 0.5\end{array}$ & $\begin{array}{l}12 \pm 0.2 \\
12 \pm 0.4 \\
14 \pm 0.3\end{array}$ & $\begin{array}{l}13 \pm 0.5 \\
13 \pm 0.3 \\
16 \pm 0.5\end{array}$ & $\begin{array}{c}74 \pm 2 \cdot 6 \\
237 \pm 17 \cdot 9 \\
284 \pm 10 \cdot 1\end{array}$ & $\begin{array}{c}58 \pm 5 \cdot 2 \\
157 \pm 18 \cdot 7 \\
179 \pm 20 \cdot 7\end{array}$ & $\begin{array}{r}110 \pm 0 \cdot 0 \\
75 \pm 5 \cdot 0 \\
25 \pm 3 \cdot 0\end{array}$ & $\begin{array}{l}21 \pm 0 \cdot 4 \\
>100 \\
>100\end{array}$ \\
\hline $\begin{array}{r}8 \\
9 \\
10\end{array}$ & $\begin{array}{l}\text { Adrenaline } \\
\mathrm{ScCl}_{3} \\
\mathrm{ScCl}_{3}+\text { adrenaline }\end{array}$ & $\begin{array}{l}4 \\
4 \\
4\end{array}$ & $\begin{array}{r}9 \pm 0.3 \\
11 \pm 0.4 \\
16 \pm 1 \cdot 1\end{array}$ & $\begin{array}{l}10 \pm 0.3 \\
11 \pm 0.4 \\
15 \pm 0.8\end{array}$ & $\begin{array}{l}12 \pm 0.4 \\
12 \pm 0.6 \\
15 \pm 0.8\end{array}$ & $\begin{array}{l}12 \pm 0.4 \\
13 \pm 0.3 \\
16 \pm 0.7\end{array}$ & $\begin{array}{c}60 \pm 3 \cdot 0 \\
171 \pm 23 \cdot 1 \\
255 \pm 17 \cdot 2\end{array}$ & $\begin{array}{c}50 \pm 1 \cdot 9 \\
66 \pm 12.9 \\
201 \pm 27 \cdot 0\end{array}$ & $\begin{array}{r}120 \pm 0 \cdot 0 \\
96 \pm 4 \cdot 0 \\
10 \pm 6 \cdot 0\end{array}$ & $\begin{array}{l}21 \pm 0 \cdot 4 \\
>100 \\
>100\end{array}$ \\
\hline
\end{tabular}


TABLE III

IN VIVO EFFECT OF SCANDIUM CHLORIDE AND ADRENALINE ON HEPARIN TOLERANCE TEST, FIBRINOGEN CONCENTRATION, THROMBOCYTE AND LEUCOCYTE COUNT

\begin{tabular}{|c|c|c|c|c|c|c|c|c|}
\hline \multirow[t]{2}{*}{ Group } & \multirow[t]{2}{*}{ Treatment } & \multirow{2}{*}{$\begin{array}{l}\text { Time of } \\
\text { Determination } \\
(h r .)\end{array}$} & \multicolumn{2}{|c|}{ Heparin Tolerance (sec.) } & \multirow{2}{*}{$\begin{array}{l}\text { Fibrinogen } \\
\text { (mg. \%) }\end{array}$} & \multirow{2}{*}{$\begin{array}{l}\text { Thrombocytes } \\
\text { (per } \mathrm{mm} .^{\mathrm{a}} \text { ) }\end{array}$} & \multirow{2}{*}{$\begin{array}{l}\text { Leucocytes } \\
\left(\text { per } m m .^{3}\right)\end{array}$} & \multirow{2}{*}{$\begin{array}{l}\text { Venous } \\
\text { Thrombosis } \\
\text { ( } \% \text { of } \\
\text { incidence) }\end{array}$} \\
\hline & & & $0.6 / i . u$ & $1 \cdot 4 / i . u$ & & & & \\
\hline 1 & None & 一 & $71 \pm 5 \cdot 5$ & $154 \pm 7 \cdot 2$ & $463 \pm 14 \cdot 3$ & $626,000 \pm 20,500$ & $8,100 \pm 460$ & 0 \\
\hline $\begin{array}{l}2 \\
3 \\
4\end{array}$ & $\begin{array}{l}\text { Adrenaline } \\
\mathrm{ScCl}_{3} \\
\mathrm{ScCl}_{3}+\text { adrenaline }\end{array}$ & $\begin{array}{l}1 \\
1 \\
1\end{array}$ & $\begin{array}{c}82 \pm 3 \cdot 1 \\
264 \pm 10 \cdot 1 \\
154 \pm 24 \cdot 2\end{array}$ & $\begin{array}{l}136 \pm 2 \cdot 6 \\
>300 \\
226 \pm 22 \cdot 1\end{array}$ & $\begin{array}{l}431 \pm 4 \cdot 8 \\
514 \pm 52 \cdot 0 \\
528 \pm 13 \cdot 2\end{array}$ & $\begin{array}{l}844,000 \pm 17,800 \\
600,000 \pm 17,500 \\
583,000 \pm 36,600\end{array}$ & $\begin{array}{l}7,000 \pm 470 \\
9,700 \pm 360 \\
9,600 \pm 400\end{array}$ & $\begin{array}{r}0 \\
0 \\
100\end{array}$ \\
\hline $\begin{array}{l}5 \\
6 \\
7\end{array}$ & $\begin{array}{l}\text { Adrenaline } \\
\mathrm{ScCl}_{3} \\
\mathrm{ScCl}_{3}+\text { adrenaline }\end{array}$ & $\begin{array}{l}2 \\
2 \\
2\end{array}$ & $\begin{array}{r}91 \pm 2 \cdot 3 \\
131 \pm 6 \cdot 1 \\
297 \pm 2 \cdot 5\end{array}$ & $\begin{array}{l}143 \pm 6 \cdot 2 \\
165 \pm 10 \cdot 2 \\
>300\end{array}$ & $\begin{array}{l}469 \pm 9 \cdot 8 \\
500 \pm 12 \cdot 8 \\
412 \pm 18 \cdot 4\end{array}$ & $\begin{array}{l}791,000 \pm 43,800 \\
703,000 \pm 11,700 \\
660,000 \pm 25,100\end{array}$ & $\begin{array}{c}7,500 \pm 510 \\
12,500 \pm 630 \\
10,700 \pm 600\end{array}$ & $\begin{array}{r}0 \\
0 \\
100\end{array}$ \\
\hline $\begin{array}{r}8 \\
9 \\
10\end{array}$ & $\begin{array}{l}\text { Adrenaline } \\
\mathrm{ScCl}_{3} \\
\mathrm{ScCl}_{3}+\text { adrenaline }\end{array}$ & $\begin{array}{l}4 \\
4 \\
4\end{array}$ & $\begin{array}{c}67 \pm 1 \cdot 2 \\
141 \pm 15 \cdot 1 \\
247 \pm 21 \cdot 3\end{array}$ & $\begin{array}{l}132 \pm 3 \cdot 2 \\
215 \pm 21 \cdot 2 \\
287 \pm 8 \cdot 8\end{array}$ & $\begin{array}{l}475 \pm 18 \cdot 6 \\
523 \pm 15 \cdot 3 \\
374 \pm 13 \cdot 5\end{array}$ & $\begin{array}{l}793,000 \pm 32,500 \\
637,000 \pm 21,200 \\
613,000 \pm 18,300\end{array}$ & $\begin{array}{c}10,700 \pm 470 \\
10,500 \pm 430 \\
11,000 \pm 480\end{array}$ & $\begin{array}{r}0 \\
0 \\
100\end{array}$ \\
\hline $\begin{array}{l}11 \\
12 \\
13\end{array}$ & $\begin{array}{l}\text { Adrenaline } \\
\mathrm{ScCl}_{\mathbf{3}} \\
\mathrm{ScCl}_{\mathbf{3}}+\text { adrenaline }\end{array}$ & $\begin{array}{l}24 \\
24 \\
24\end{array}$ & $\begin{array}{r}83 \pm 2 \cdot 2 \\
62 \pm 5 \cdot 2 \\
122 \pm 4 \cdot 7\end{array}$ & $\begin{array}{l}154 \pm 6 \cdot 4 \\
148 \pm 8 \cdot 9 \\
170 \pm 7 \cdot 3\end{array}$ & $\begin{array}{l}646 \pm 16 \cdot 0 \\
603 \pm 13 \cdot 5 \\
784 \pm 9 \cdot 7\end{array}$ & $\begin{array}{l}797,000 \pm 23,900 \\
503,000 \pm 12,700 \\
387,000 \pm 29,500\end{array}$ & $\begin{array}{r}8,900 \pm 600 \\
8,900 \pm 600 \\
11,000 \pm 760\end{array}$ & $\begin{array}{r}0 \\
0 \\
100\end{array}$ \\
\hline
\end{tabular}

content $(\mathrm{P}<0.001)$ (Table II), heparin tolerance $(P<0.001)$, and fibrinogen concentration $(P<$ 0.001 ) (Table III). In the groups killed after 24 hours there was a significant difference only in thrombocyte count $(\mathbf{P}<0.001)$ (Table III).

Massive mixed thrombi of the femoral vein (propagating into the iliac vein and sometimes into the vena cava) were found in all animals given scandium chloride and adrenaline (Table III). Stasis and thrombosis were also observed in the small tributary veins.

\section{DISCUSSION}

The most striking result of these experiments is that thrombus formation can be produced in the presence of impaired blood coagulability.

It has been proven that the first step in the formation of white thrombi is the adhesion and aggregation of thrombocytes at a site of endothelial damage (Hayem, 1878; Owren, Hellem, and Ödegaard, 1964). During phylogenesis this cellular defensive reaction developed before the mechanism for blood coagulation which is bound to plasma factors (Loeb, 1906; Silberberg, 1938); it functions even in the state of hypocoagulability (Loeb, 1906; Zucker, 1947). Some coagulation factors can be adsorbed and concentrated on the surface of thrombocytes even from a plasma in which they are scarce (Bounameaux, 1955; Owren, 1962). It has also been found that anticoagulants enhance platelet deposition at the site of endothelial injury (Fulton, Akers, and Lutz, 1953). Therefore, it is understandable that experimental and spontaneous thrombus formation can occur even in a state of hypocoagulability. This conclusion agrees with the view that blood coagulation and thrombosis are different and usually not interdependent processes (Erichson, 1965).

The results of our experiments raise doubts about the prognostic value of routine coagulation tests and show that during the development of thrombi, there appear signs of a consumption coagulopathy.

This work was supported by the Canadian Heart Foundation and the Medical Research Council of Canada.

\section{REFERENCES}

Bounameaux, Y. (1955). C. R. Soc. Biol. (Paris), 149, 1285.

Egeberg, O., and Owren, P. A. (1963). Brit. med. J., 1, 220.

Erichson, R. B. (1965). N.Y. State J. Med., 65, 1091.

Evans, W. (1954). Proc. roy. Soc. Med., 47, 318.

Fulton, G. P., Akers, R. P., and Lutz, B. R. (1953). Blood, 8, 140.

Gabbiani, G., Jacqmin, M. L., and Selye, H. (1966). J. Pharmacol. exp. Ther., in the press.

Hayem, G. (1878). Arch. Physiol. norm. path., 5, 692.

Langdell, R. D., Wagner, R. H., and Brinkhous, K. M. (1953). J. Lab. clin. Med., 41, 637.

Loeb, L. (1906). Virchows Arch. path. Anat., 185, 160.

O'Neill, J. F. (1947). Ann. Surg., 126, 270.

Owen, C. A., Jr., Mann, F. D., Hurn, M. M., and Stickney, J. M. (1955). Amer. J. clin. Path., 25, 1417.

Owren, P. A. (1962). Proc. 8th int. Congr. Haemat., 3, 1522.

, Hellem, A. J., and Ödegaard, A. (1964). Lancet, 2, 975.

Perlick, E. (1960). Gerrinunngslaboratorium in Klinik und Praxis. Thieme, Leipzig.

Pechet, L., and Alexander, B. (1961). New Engl. J. Med., 265, 1093.

Quick, A. J. (1935). J. biol. Chem., 109, 73 P.

- . (1958). Surg. Clin. N. Amer., 38, 1031.

Rodman, N. F., Jr., Mason, R. G., and Brinkhous, K. M. (1963). Fed. Proc., 22, 1356.

Selye, H., and Tuchweber, B. (1964). Allergie u. Asthma, 10, 335.

,-- , and Rohan, P. (1965). Nature (Lond.), 208, 900.

Silberberg, M. (1938). Physiol. Rev., 18, 197.

Sirridge, M. S., Bowman, K. S., and Allwin, J. F. (1962). Amer. J. clin. Path., 37, 551.

Soulier, J. P. (1951). Sem. Hôp. Paris, 27, 775.

Virchow, R. (1856). Gesammelte Abhandlungen zur wissenschaftlichen Medicin. von Meidinger, Frankfurt.

Wessler, S. (1963). Fed. Proc., 22, 1366.

Zweifach, B. W. (1963). Ibid., 22, 1351.

Zucker, M. B. (1947). Amer. J. Physiol., 148, 275. 